\title{
THE SIMILARITY SOLUTION FOR NONLINEAR PARABOLIC EQUATION
}

\author{
M.E. KHALIFA, M.S. SLEEM AND H.A. ZEDAN
} Abstract. We study the properties of Lie group and investigate the similarities
of the flow of ground water equation in the form

$$
h_{t}=\frac{k}{2 m}\left[h_{y y}^{2}+h_{x x}^{2}\right]
$$

\section{Introduction}

In the study of partial differential equations, the discovery of explicit solutions has great theoretical and practical importance. In case of linear systems, general solutions can be built up by superposition from separable solutions; for nonlinear systems, explicit solutions are used as models for physical or numerical experiments, and often reflect the asymptotic behavior of more complicated solutions. Over the years, a variety of methods for finding these special solutions by reducing the partial differential equations to one or more ordinary differential equations have been deviced (see [1], [7], [8], [10]).

In this paper we investigate the similarity for the flow of ground water [9] which was formulated by Forchheimer as follows:

$$
\frac{\partial h}{\partial t}=\frac{k}{2 m}\left[\frac{\partial^{2} h^{2}}{\partial x^{2}}+\frac{\partial^{2} h^{2}}{\partial y^{2}}\right]
$$

where $h(t, x, y)$ is an unknown function which represents the ground water depth, $m$ represents the porosity and $k$ is a constant. It is obvious that equation (1.1) is nonlinear in $h$ and is accordingly much more difficult to handle.

Received Febuary 10, 1993.; revised June 30, 1993.

1991 Mathematics Subject Classification. 35K55, 35Q35, 17 B81.

Key words and phrases. Nonlinear parabolic equation, similarity solution, flow of ground water. 
First, we will study the properties of Lie group and algebra associated with equation (1.1) (see [1], [6]). Second, we will investigate a new method of deriving similarities of partial differential equations which has recently been developed by [4]. The unusual characteristics about this method in comparison with the other [2], [3] is that it involves no use of group theory. This method has been successfully applied to obtain new similarity solution for several problems (See [5]).

\section{The Properties of Lie Group}

In equation (1.1) let $h^{2}=u$ and $\frac{k}{m}=S$. We get

$$
H u \equiv-u_{t}+S \sqrt{u}\left(u_{x x}+u_{y y}\right)=0
$$

Let

$$
\begin{aligned}
v & =\zeta(x, y, t, u) \frac{\partial}{\partial x}+\eta(x, y, t, u) \frac{\partial}{\partial y}+\tau(x, y, t, u) \frac{\partial}{\partial t} \\
& +\phi(x, y, t, u) \frac{\partial}{\partial u}
\end{aligned}
$$

be a vector field. We wish to determine all possible coefficient functions $\zeta, \eta, \tau, \phi$. According to the theorem 2.31. in [11], we need to know the second prolongation which is represented by

$$
p r^{2} v=v+\phi^{x} \frac{\partial}{\partial u_{x}}+\phi^{y} \frac{\partial}{\partial u_{y}}+\phi^{t} \frac{\partial}{\partial u_{t}}+\phi^{x x} \frac{\partial}{\partial u_{x x}}+\phi^{y y} \frac{\partial}{\partial u_{y y}}
$$

We can apply the equation (2.2), (2.3) for the equation (2.1) to get

$$
\begin{gathered}
v(H u)=-\frac{S}{2} \frac{\phi(x, y, t, u)}{\sqrt{u}}\left(u_{x x}+u_{y y}\right) \\
p r^{2} v(H u)=v(H u)+\phi^{t}-S \sqrt{u} \phi^{x x}-S \sqrt{u} \phi^{y y}=0
\end{gathered}
$$

Putting $\phi^{i}, \phi^{x x}, \phi^{y y}$ (See [11]) in (2.5) and equating the coefficients of various monomials in the first and second order partial derivatives of $u$, we get the most general infinitesimal symmetry of (2.1) in the form

$$
\begin{aligned}
\zeta & =\frac{1}{2} A x-B y+E, \quad \eta=\frac{1}{2} A y+B x+C \\
\tau & =A t+D, \quad \phi=0 .
\end{aligned}
$$

It is easy to show that the Lie algebra of infinitesimal symmetries of equation (2.1) is spanned by five vector fields as follows

$$
V_{1}=\frac{1}{2} x \partial_{x}+\frac{1}{2} y \partial_{y}+t \partial_{t}, \quad V_{2}=-y \partial_{x}+x \partial_{y}
$$




$$
V_{3}=\partial_{y}, \quad V_{4}=\partial_{x}, \quad V_{5}=\partial_{t}
$$

Then the solution of equation (2.1) maps into itself, where this equation is invariant under the group of transformation

$$
\begin{aligned}
& \bar{x}=x+\varepsilon \zeta(x, y)+O\left(\varepsilon^{2}\right) \\
& \bar{y}=y+\varepsilon \eta(x, y)+O\left(\varepsilon^{2}\right) \\
& \bar{t}=t+\varepsilon \tau(t)+O\left(\varepsilon^{2}\right) \\
& \bar{u}=u+O\left(\varepsilon^{2}\right)
\end{aligned}
$$

Then the characteristic equations are:

$$
\begin{gathered}
\frac{d x}{\zeta}=\frac{d y}{\eta}=\frac{d t}{\tau}=\frac{d u}{\phi} \\
\frac{d x}{\frac{1}{2} A x-B y+E}=\frac{d y}{\frac{1}{2} A y+B x+C}=\frac{d t}{A t+D}=\frac{d u}{O}
\end{gathered}
$$

We solve these equations to obtain the similarity transform which can be represented as follows:

$$
\begin{aligned}
\zeta_{1} & =\frac{\left(\frac{1}{2} A x-B y+E\right)^{2}}{A t+D} \\
\eta_{1} & =\frac{\left(\frac{1}{2} A y+B x+C\right)^{2}}{A t+D} . \\
u & =F\left(\zeta_{1}, \eta_{1}\right)
\end{aligned}
$$

Using (2.7) and (2.1) we get

$$
H_{1}(F) \equiv \frac{S\left(A^{2}+4 B^{2}\right)}{A}\left\{\zeta_{1} \sqrt{F} F_{\zeta_{1} \zeta_{1}}+\eta_{1} \sqrt{F} F_{\eta_{1} \eta_{1}}+\frac{1}{2} \sqrt{F} F_{\zeta_{1}}+\frac{1}{2} \sqrt{F} F_{\eta_{1}}\right\}+\zeta_{1} F_{\zeta_{1}}+\eta_{1} F_{\eta_{1}}=0
$$

It is obvious that the similarity transform reduces the independent variables by one; applying to equation (2.8) the same algorithim previously applied to equation (2.1) where

$$
\begin{gathered}
V=X\left(\zeta_{1}, \eta_{1}, F\right) \frac{\partial}{\partial \zeta_{1}}+Y\left(\zeta_{1}, \eta_{1}, F\right) \frac{\partial}{\partial \eta_{1}}+Z\left(\zeta_{1}, \eta_{1}, F\right) \frac{\partial}{\partial F} \\
p r^{2} V=V+Z^{\zeta_{1}} \frac{\partial}{\partial F_{\zeta_{1}}}+Z^{\eta_{1}} \frac{\partial}{\partial F_{\eta_{1}}}+Z^{\zeta_{1} \zeta_{1}} \frac{\partial}{\partial F_{\zeta_{1} \zeta_{1}}}+Z^{\eta_{1} \eta_{1}} \frac{\partial}{\partial F_{\eta_{1} \eta_{1}}}
\end{gathered}
$$

we get:

$$
\begin{aligned}
V\left(H_{1} F\right)= & \left(L \sqrt{F} F_{\zeta_{1} \zeta_{1}}+F_{\zeta_{1}}\right) X+\left(L \sqrt{F} F_{\eta_{1} \eta_{1}}+F_{\eta_{1}}\right) Y \\
& +\frac{1}{2} \frac{Z L}{\sqrt{F}}\left(\zeta_{1} F_{\zeta_{1} \zeta_{1}}+\eta_{1} F_{\eta_{1} \eta_{1}}+\sqrt{F} F_{\zeta_{1}}+\sqrt{F} F_{\eta_{1}}\right)
\end{aligned}
$$




$$
\begin{aligned}
\operatorname{pr}^{2} V\left(H_{1} F\right)= & L \sqrt{F} F_{\zeta_{1} \zeta_{1}} x+F_{\zeta_{1}} X+L \sqrt{F} F_{\eta_{1} \eta_{1}} Y+F_{\eta_{1}} Y \\
& +\frac{L}{2} Z_{\zeta_{1}} F-\frac{1}{2} F_{\zeta_{1} \zeta_{1}}+\frac{L}{2} Z_{\eta_{1}}-\frac{L}{2} F_{\eta_{1} \eta_{1}}+\frac{L}{2} Z F_{\zeta_{1}}+\frac{L}{2} Z F_{\eta_{1}} \\
& +\frac{L}{2} Z^{\zeta_{1}} \sqrt{F}+\zeta_{1} Z^{\zeta_{1}}+\frac{L}{2} Z^{\eta_{1}} \sqrt{F}+\eta_{1} Z^{\eta_{1}} \\
& +L \zeta_{1} \sqrt{F} Z^{\zeta_{1} \zeta_{1}}+L \eta_{1} \sqrt{F} Z^{\eta_{1} \eta_{1}}=0
\end{aligned}
$$

and $L=\frac{S\left(A^{2}+4 B^{2}\right)}{A}$. Substituting the values of $Z^{\zeta_{1}}, Z^{\eta_{1}}, Z^{\zeta_{1} \zeta_{1}}, Z^{\eta_{1} \eta_{1}}$ in (2.9) and equating the coefficients of the various monomial in the first and second order partial derivatives of $F$, we get the infinitesimal symmetry of (2.9) in the form,

$$
X=D \sqrt{\zeta}_{1} \quad, \quad Y=C \sqrt{\eta}_{1} \quad, \quad Z=0
$$

and hence the characteristic equation

$$
\frac{d \zeta_{1}}{X}=\frac{d \eta_{1}}{Y}=\frac{d F}{Z}
$$

gives the following similarity transform

$$
\begin{aligned}
\theta & =2 C \sqrt{\zeta}_{1}-2 D \sqrt{\eta}_{1} \\
F & =\psi(\theta)
\end{aligned}
$$

Substituting (2.11) into (2.8) we get the following ordinary differential equation

$$
L\left(C^{2}+D^{2}\right) \sqrt{\psi} \psi^{\prime \prime}(\theta)+\theta \psi^{\prime}(\theta)=0
$$

where $\mathrm{L}, \mathrm{C}, \mathrm{D}$ are constants.

\section{New Similarity}

In this section we seek a reduction of the equation (2.1) to the form:

$$
u(x, y, t)=\alpha(x, y, t)+\beta(x, y, t) w(z(x, y, t))
$$

where $\alpha(x, y, t), \beta(x, y, t)$ and $z(x, y, t)$ are to be determined. First we prove that it is sufficient to seek similarity reduction of equation (2.1) in the form (3.1) rather than the more general form:

$$
u(x, y, t)=U(x, y, t, w(z(x, y, t))
$$

which is the most general form for similarity reduction (cf. Bluman and cole [9]). Substituting (3.1) into (2.1) and collecting coefficients of monomials of $W$ and its derivatives yields:

$$
\begin{aligned}
& S \sqrt{U}\left[W^{\prime \prime}\left(U_{w} z_{x}^{2}+U_{w} z_{y}^{2}\right)+W^{\prime^{2}}\left(U_{w w} z_{x}^{2}+U_{w w} z_{y}^{2}\right)\right. \\
& +W^{\prime}\left(2 U_{w x} z_{x}+2 U_{w y} z_{y}+U_{w} z_{x x}+U_{w} z_{y y}-U_{w} z_{t}\right)+U_{x x} \\
& \left.+U_{y y}-U_{t}\right]=0
\end{aligned}
$$




$$
S=\frac{K}{m},\left(^{\prime}\right)=\frac{d}{d z}
$$

Remark 1. We use the coefficient of $W^{\prime \prime}$ as the normalizing coefficient and therefore require that the other coefficient be of the form:

$$
U_{w}\left(z_{x}^{2}+z_{y}^{2}\right) \Gamma(z, w)
$$

where $\Gamma$ is a function to be determined.

Remark 2. We reserve uppercase greek letters for undetermined functions of $z$ so that after, performing operations (differentiation, integration, exponentiation, rescaling, etc.) the result can be denoted by the same letter [e.g., the derivative of $\Gamma(z)$ will be called $\Gamma(z)]$.

Remark 3. There are three freedoms in the determination of $\alpha, \beta, z$ and $w$ we can exploit, without loss of generality, that are valuable in keeping the method manageable: (i) if $\alpha(x, t)$ has the form $\alpha=\alpha_{o}(x, t)+\Omega(z) \beta(x, t)$ then we can take $\Omega \equiv 0$ [by subsituting $w(z) \rightarrow w(z)-\Omega(z)]$; (ii) if $\beta(x, t)$ has the form $\beta=\beta_{o}(x, t) \Omega(z)$, then we can take $\Omega \equiv 1$ [by substituting $w(z) \rightarrow w(z) / \Omega(z)$ ]; and (iii) if $z(x, t)$ is determined by an equation of the form $\Omega(z)=z_{o}(x, t)$, where $\Omega(z)$ is any invertible function, then we can take $\Omega(z)=z$ [by substituting $z \rightarrow \Omega^{-1}(z)$ ].

Using the coefficient of $w^{\prime \prime}$ as normalizing coefficient, then the coefficient of $w^{\prime^{2}}$ requires that:

$$
S \sqrt{U} U_{w}\left(z_{x}^{2}+z_{y}^{2}\right) \Gamma(z, w)=S \sqrt{U} U_{w w}\left(z_{x}^{2}+z_{y}^{2}\right)
$$

and hence

$$
\Gamma(z, w)=\frac{U_{w w}}{U_{w}}
$$

Integrating twice and using remark (3) we get $U(x, y, t, w)=\theta(x, y, t) \Gamma(w, z)+\phi(x, y, t)$ where $\theta, \phi$ arbitrary functions.

Therefore it is sufficient to seek similarity reduction of equation (2.1) in the form (3.1). After putting (3.1) into (2.1) and taking $z_{y}=0$ we can get the following:

$$
\begin{aligned}
0= & -\alpha_{i}^{2}+s^{2} \alpha \alpha_{x x}+s^{2} \alpha \alpha_{y y}^{2}+2 s^{2} \alpha_{x x} \alpha_{y y}+w^{\prime}\left[-2 \alpha_{t} \beta Z_{t}\right. \\
& \left.+4 s^{2} \alpha \alpha_{x x} \beta_{x} Z_{x}+2 s^{2} \alpha \alpha_{x x} \beta Z_{x x}+4 s^{2} \alpha \alpha_{y y} \beta_{x} Z_{x}+2 s^{2} \alpha \beta \alpha_{y y} Z_{x x}\right] \\
& +w\left[-2 \alpha_{i} \beta_{t}+2 s^{2} \alpha \alpha_{x x} \beta_{x x}+2 s^{2} \alpha \alpha_{y y} \beta_{y y}+2 s^{2} \alpha \alpha_{x x} \beta_{y y}\right. \\
& \left.+2 s^{2} \alpha \alpha_{y y} \beta_{x x}+s^{2} \beta \alpha_{x x}^{2}+s^{2} \beta \alpha_{y y}^{2}+2 s^{2} \beta \alpha_{x x} \alpha_{y y}\right] \\
& +w^{\prime^{2}}\left[-\beta^{2} Z_{t}^{2}+4 s^{2} \alpha \beta_{x}^{2} Z_{x}^{2}+s^{2} \alpha \beta^{2} Z_{x x}^{2}+4 s^{2} \alpha \beta \beta_{x} Z_{x} Z_{x x}\right] \\
& +w^{2}\left[-\beta^{2}+s^{2} \alpha \beta_{x x}^{2}+2 s^{2} \alpha \beta_{x x}^{2} \beta_{y y}+2 s^{2} \alpha_{x x} \beta \beta_{x x}+2 s^{2} \alpha_{y y} \beta \beta_{y y}\right. \\
& \left.+2 s^{2} \alpha_{x x} \beta \beta_{y y}+2 s^{2} \alpha_{y y} \beta \beta_{x x}+s^{2} \alpha \beta_{y y}^{2}\right]+w w^{\prime}\left[-2 \beta \beta_{t} Z_{t}\right. \\
& +2 s^{2} \alpha \beta \beta_{x x} Z_{x x}+4 s^{2} \alpha \beta_{x} \beta_{x x} Z_{x}+4 s^{2} \alpha \beta_{x} \beta_{y y} Z_{x}+2 s^{2} \alpha \beta \beta_{y y} Z_{x x}
\end{aligned}
$$




$$
\begin{aligned}
& \left.+4 s^{2} \beta \beta_{x} Z_{x} \alpha_{x x}+2 s^{2} \alpha_{x x} \beta^{2} Z_{x x}+4 s^{2} \alpha_{y y} \beta \beta_{x} Z_{x}+2 s^{2} \beta^{2} \alpha_{y y} Z_{x x}\right] \\
& +w^{\prime \prime}\left[s^{2} \alpha \beta^{2} Z_{x}^{4}\right]+w^{\prime} w^{\prime \prime}\left[2 s^{2} \alpha \beta^{2} Z_{x}^{2} Z_{x x}+4 s^{2} \alpha \beta \beta_{x} Z_{x}^{3}\right] \\
& +w w^{\prime \prime}\left[2 s^{2} \alpha \beta \beta_{x x} Z_{x}^{2}+2 s^{2} \beta^{2} \alpha_{x x} Z_{x}^{2}+2 s^{2} \alpha_{y y} \beta^{2} Z_{x}^{2}+2 s^{2} \alpha \beta \beta_{y y} Z_{x}^{2}\right] \\
& +w^{\prime \prime}\left[2 s^{2} \alpha \alpha_{x x} \beta Z_{x}^{2}+2 s^{2} \alpha \alpha_{y y} \beta Z_{x}^{2}\right]+w w^{\prime 2}\left[4 s^{2} \beta \beta_{x}^{2} Z_{x}^{2}\right. \\
& \left.+s^{2} \beta^{3} Z_{x x}^{2}+4 s^{2} \beta^{2} \beta_{x} Z_{x} Z_{x x}\right]+w w^{\prime \prime}\left[s^{2} \beta^{3} Z_{x}^{4}\right]+w w^{\prime} w^{\prime \prime}\left[2 s^{2} \beta^{3} Z_{x}^{2} Z_{x x}\right. \\
& \left.+4 s^{2} \beta^{2} \beta_{x} Z_{x}^{3}\right]+w^{2} w^{\prime \prime}\left[2 s^{2} \beta^{2} \beta_{x x} Z_{x}^{2}+2 s^{2} \beta^{2} \beta_{y y} Z_{x}^{2}\right]+w^{3}\left[s^{2} \beta \beta_{x x}^{2}\right. \\
& \left.+s^{2} \beta \beta_{y y}^{2}+2 s^{2} \beta \beta_{x x} \beta_{y y}\right]+w^{2} w^{\prime}\left[2 s^{2} \beta^{2} \beta_{x x} Z_{x x}+4 s^{2} \beta \beta_{x} \beta_{x x} Z_{x}\right. \\
& \left.+4 s^{2} \beta \beta_{x} \beta_{y y} Z_{x}+2 s^{2} \beta^{2} \beta_{y y} Z_{x x}\right] .
\end{aligned}
$$

We can take the coefficient of $w w^{\prime \prime^{2}}$ as a normalizing coefficient. Then coefficient $w^{\prime \prime^{2}}$ becomes:

$$
s^{2} \alpha \beta^{2} Z_{x}^{4}=s^{2} \beta^{3} Z_{x}^{4} \Gamma(z)
$$

and hence

$$
\alpha=\beta \Gamma(z)
$$

Therefore, using Remark (3), we get

$$
\alpha=0
$$

Substituting (3.5) into (3.4) we get:

$$
\begin{aligned}
& w^{\prime^{2}}\left[-\beta^{2} Z_{t}^{2}\right]+w^{2}\left[-\beta^{2}\right]+w w^{\prime}\left[-2 \beta \beta_{t} Z_{t}\right]+w w^{\prime \prime^{2}}\left[s^{2} \beta^{3} Z_{x}^{4}\right] \\
& +w w^{\prime^{2}}\left[4 s^{2} \beta \beta_{x}^{2} Z_{x}^{2}+s^{2} \beta^{3} Z_{x x}^{2}+4 s^{2} \beta_{x} Z_{x} Z_{x x}\right] \\
& +w w^{\prime} w^{\prime \prime}\left[2 s^{2} \beta^{3} Z_{x}^{2} Z_{x x}+4 s^{2} \beta^{2} \beta_{x} Z_{x}^{3}\right]+w^{2} w^{\prime \prime}\left[2 s^{2} \beta^{2} \beta_{x x} Z_{x}^{2}\right. \\
& \left.+2 s^{2} \beta^{2} \beta_{y y} Z_{x}^{2}\right]+w^{3}\left[s^{2} \beta \beta_{x x}^{2}+s^{2} \beta \beta_{y y}^{2}+2 s^{2} \beta \beta_{x x} \beta_{y y}\right] \\
& +w^{2} w^{\prime}\left[2 s^{2} \beta^{2} \beta_{x x} Z_{x x}+4 s^{2} \beta \beta_{x} \beta_{x x} Z_{x}+4 s^{2} \beta \beta_{x} \beta_{y y} Z_{x}\right. \\
& +2 s^{2} \beta^{2} \beta_{y y} Z_{x x}=0
\end{aligned}
$$

Since the coefficients of $w^{\prime 2}$ and $w^{2}$ yield

$$
-\beta^{2} Z_{t}^{2} \Gamma(z)=-\beta^{2}
$$

Integrating and using Remark 3 it is easy to show that:

$$
Z=t+b, \quad(b=\text { const. })
$$

Also the coefficients of $w^{\prime 2}$ and, $w w^{\prime}$ yield

$$
-\beta^{2} Z_{t}^{2} \Gamma(z)=-2 \beta \beta_{t} Z_{t}
$$

Using Remark (3) and integrating, we obtian

$$
\beta=\phi(x)
$$


where $\phi(y, t)$ is any arbitrary function, using (3.5), (3.7), (3.8), equation (3.6) becomes

$$
-\phi^{2}\left(w^{2}+w^{\prime^{2}}\right)+w^{3}\left(s^{2} \phi \phi_{x x}^{2}\right)=0
$$

This is an ordinary differential equation for $w(z)$ provided that

$$
\phi^{2} \gamma(z)=\phi \phi_{x x}^{2}
$$

where $\gamma(z)$ are to be detemined. Since $Z=t+b$, necessarily $\gamma(z)=A$ constant. Hence, equation (3.9) becomes

$$
A s^{2} \phi^{2} w^{3}-\left(w^{\prime 2}+w^{2}\right)=0
$$

Therefore the general similarity reduction of equation $(2.1)$ is given by $u(x, y, t)=$ $\phi(z) w(z), Z=t+b, b$ is constant.

\section{References}

[1] W. F. Ames, Nonlinear partial Differnetial Equations in Engineering, Academic press, New york, 1965.

[2] G. W. Bluman, Similarity Methods for Differential Equations, Springer-Verlage, New York, 1974.

[3] G. W. Bluman, On the transformation of diffusion processes into the wiener process.

[4] P. A. Clarkson, M. D. Kruskal, "New similarity reductions of Boussinesq equation," J. Math. phys., 30 (10), October 1989.

[5] P. A. Clarkson, "New exact solutions of the Boussinesq equation" Euro. Jn of Applied Mathematics, (1990) Vol. 1 pp. 279-300.

[6] S. Hasn, "Differential Equations, their solution using symmetries", Cambridge University press 1989.

[7] A. G. Hansen, "Similarity anlysis of boudary value problems in engineering", Prentice-Hall, Englewood Cliffs, New Jersey, 1964.

[8] P. J. Olver, P. Rasenau, "The construction of special solutions to partial differential equations," Physics Letters, volume 114A, No. 3 1986, pp. 107-112.

[9] H. Rouse, "Engineering hydraulics ," Proceedings of the fourth hydraulics conference, Iowa institute of hydraulic research, June, pp. 12-15, 1949, pp. 383-385.

[10] R. Seshadri, T. Y. Na, Group invariance in engineering boundary value problenes, Springer-verlag, 1985.

[11] P. J. Olver, Applications of Lie groups to differential equations, Springer New York, 1986.

Mathematics Department, Faculty of Education, Kafr El-Sheikh, Egypt. 